\title{
Spin-Dependent Effects in Helical Molecular Systems with Rashba-Like Spin-Orbit Interaction
}

\author{
R. Gutierrez ${ }^{a}$ And G. CUniberti ${ }^{a, b}$ \\ ${ }^{a}$ Institute for Materials Science, Dresden University of Technology, 01062 Dresden, Germany \\ ${ }^{b}$ Dresden Center for Computational Materials Science and Center for Advancing Electronics Dresden; \\ Dresden University of Technology, 01062 Dresden, Germany
}

\begin{abstract}
Strong spin-selective effects have been recently observed in both photoemission and electrical transport experiments in biomolecular systems, opening fascinating possibilities for interfacing semiconductor and biomolecular systems to create highly efficient spintronics devices. From the theoretical and experimental point of view there are strong suggestions that molecular chirality is playing a crucial role. In this study we extend a previously formulated model (R. Gutierrez, E. Díaz, R. Naaman, G. Cuniberti, Phys. Rev. B 85, 081404 (2012)) describing the linear propagation of a charge with spin along the axis of a helical charge distribution. We explore different parameter regions and show that a strong negative spin polarization as observed in the previously mentioned experiments can be obtained with reasonable values of both the electronic coupling elements and the helical field induced spin-orbit interaction.
\end{abstract}

DOI: $10.12693 /$ APhysPolA.127.185

PACS: 73.22.-f, 73.63.-b, 72.25.-b, 87.14.gk, 87.15.Pc

\section{Introduction}

Exploiting the spin degree of freedom to transfer information or perform logic operations builds the basis of spintronics. Currently, the majority of existing spintronic devices are based on inorganic materials. However, using organic molecules in spintronics, although very challenging, would offer many advantages such as the possibility for chemically tuning the spin-dependent response as well as their inexpensive synthesis in large amounts. Organic molecules have been already proposed for being used e.g. as spin-valves [1-5], and spin dependent tunneling through organic molecules adsorbed on magnetic substrates has been theoretically demonstrated [6]. In general terms, the spin selectivity in organic-based spintronics devices is rather related to the intrinsic magnetic properties of the electrodes or of that of the molecules. For molecules lacking magnetic properties, no strong spin-dependent properties might be expected. Surprinsingly, it has been recently shown experimentally $[7,8]$ that monolayers of double-stranded DNA (dsDNA) oligomers display strong spin selectivity. These results can have deep implications not only for the design of novel, bio-inspired spintronic devices, but also shed a new light on electron transfer in biologically relevant molecules. Meanwhile, first devices based on this high spin selectivity have been demonstrated: a chiralbased magnetic-based silicon-compatible memory device without a permanent magnet [9]. Spin-selective charge transfer through a self-assembled monolayer (SAM) of polyalanine was hereby used to magnetize a Ni layer with a magnetization that corresponds to applying an external magnetic field of the order of $0.4 \mathrm{~T}$ to the Ni layer [9].

The problem of spin sensitivity in SAMs started to draw attention already in the late 90s. Thus, Ray et al. found in 1999 that organized organic films of the chiral molecule L- or D-stearoyl lysine showed a large asymmetry in the scattering probability of spin up and spin down polarized electrons [10]. The found asymmetries were considerably higher than in gas-phase experiments. Later on, Carmeli et al. reported similar results for self-assembled monolayers of polyalanine polypeptides [11]. Several other experiments have meanwhile showed the same spin-filter-effect for different systems [12-16]. However, a breakthrough took place in 2011, when the two previously mentioned new experiments $[7,8]$ were published. In the first set of experiments [7], spin-selective transmission of unbounded electrons through a SAM of dsDNA on gold was demonstrated. Hereby, polarized light was used to eject photoelectrons with a definite spin polarization state from the metal surface. By directly measuring the spin of the transmitted electrons with a Mott polarimeter, values of the spin polarization as high as $60 \%$ at room temperature were found. More interestingly, the spin-polarized photoelectrons were detected even when the photoelectrons were generated with unpolarized light. Furthermore, a linear increase of the spin polarization with increasing thickness (i.e. length of the dsDNA molecules) of the SAM was observed. In a second type of experiments [8], a SAM of single-strand (ss) DNA was deposited on a nickel film on top of a Si substrate. An AFM tip functionalized with a gold nanoparticle to which a ssDNA was attached with a sequence complementary to that of the SAM, was approached to the SAM until a molecular junction was created by the hybridization of the two complementary strands. Ideally, the junction consists of a single dsDNA molecule, so that the probed electrical response is not that of the whole monolayer. A permanent magnet underneath the nickel substrate controlled the electronic spin alignment in the nickel. The measured $I-V$ characteristics of the molecular junction revealed spin selectivity, the effect being very pronounced for the 40 and 50 base pairs oligomers and much less pronounced in the case of shorter, 26 base pairs long, 
molecules. Recent photoemission experiments on bacteriorhodopsine [17] along the lines of Ref. [7], seem to demonstrate that (i) the phenomenon of spin selectivity is rather universal and (ii) a double-strand structure of the molecular system is not required to have the effect.

Concerning now the theoretical approaches, there have been only few investigations directly related to the spin selectivity. Common to all theoretical attempts is to postulate that there is a sensitive interplay between chirality and spin-selectivity. More specifically, most of the proposed models assume that an electrostatic potential with helical symmetry induces a spin-orbit coupling (SOC) term, which can then affect the propagation of the electron through the system. To our knowledge there have been two main theoretical lines to approach the problem, which correlate with the two main types of experimental approaches mentioned above: (i) Studies based on scattering theory at the level of the Born approximation [18, 19] including spin-orbit interactions and a helical potential. This approach can be closely related to the experiments in Ref. [7], where the energies of the emitted electrons lie well above the energy of molecular orbitals of the DNA molecules and thus, the problem can be viewed as a scattering process in an external helical potential; (ii) Approaches based on quantum transport $[20,25]$ have been also proposed, being closer related to the second class of experiments [8], which probe the electrical response of dsDNA molecular junctions in a two terminal setup. Reference [20] addressed for the first time in the context of a quantum transport model the possibility that an electrostatic field with helical symmetry could induce a spin-orbit interaction and the consequences for charge transport. In Ref. [22], the motion of a charge along a helical path including spin-orbit interactions was treated within a tight-binding model. A sizeable spin polarization was found. In Ref. [21] a consistent model Hamiltonian was then derived, describing the propagation of a spin along a helical path and with full inclusion of an external helical potential generated by an array of point charges. It was demonstrated that in this model a strong spin selectivity was possible, if an orbital-dependent spin-orbit coupling was assumed. In Ref. [23] a different mechanism to explain the observed polarization has been proposed. The authors introduced the concept of induced spin filtering, where selectivity in the transmission of the electron orbital angular momentum would induce spin selectivity in the transmission process in case that the metal substrate displayed strong spin-orbit coupling. In this sense, the observed polarization turns out to be conditioned by the strong spin-orbit coupling in the substrate rather than by a strong SOC inside the molecular system. This effect seems however only to be important for e.g. gold substrates with strong SOC, but not for surfaces with a much weaker SOC interaction.

In the current study, we extend the model of Ref. [20] to include more than one orbital per lattice site in the tight-binding representation of the model (see next section). We keep however the main assumption of Ref. [20], where a charge was considered to move with only $z$-component of its momentum (along the helical axis). This allows to contrast the results with the investigations in Ref. [21], where a helical path with a finite curvature radius and torsion was assumed.

\section{Model Hamiltonian and transport approach \\ 2.1. Model Hamiltonian}

Our starting point is that in a helical molecular system like DNA, the electrostatic potential distribution should display helical symmetry. As a result, a charge moving along the helical system will experience in its rest frame an effective, momentum-dependent magnetic field which can couple to its spin, leading to a spin-orbit interaction contribution. Thus, if the momentum of the charge is $\boldsymbol{p}$ and we denote the helical field by $\boldsymbol{E}_{\text {helix }}$, the SOC Hamiltonian becomes $H_{\mathrm{SO}}=\lambda \boldsymbol{\sigma}\left(\boldsymbol{p} \times \boldsymbol{E}_{\text {helix }}\right)$. The SOC strength is $\lambda=e \hbar / 4 m^{2} c^{2}$ and $\boldsymbol{\sigma}$ is a vector whose components are the Pauli spin matrices $\sigma_{x}, \sigma_{y}$, $\sigma_{z}$. Although this term includes a full three-dimensional motion of the charge, we will assume from now that the motion takes place along the helical axis, so that $p_{x}=p_{y}=0, p_{z} \neq 0$. We model the electrostatic field in a simple way by assuming a distribution of point charges along a helix $[20,26]$. The general solution is quite involved, but the assumption of linear motion near the helical axis allows to use approximate expressions for the $x, y$ field components (the $z$-component does not play any role by the assumption of linear motion along the $z$-axis):

$$
\begin{aligned}
& \boldsymbol{E}_{\text {helix }}(z)=\left(E_{x}(z), E_{y}(z), 0\right)= \\
& \quad-E_{0} \sum_{i, j} g_{i j}(z)(\cos (Q j \Delta z), \sin (Q j \Delta z), 0) .
\end{aligned}
$$

Here, the geometric factor $g_{i j}(z)=(1+[(z-\boldsymbol{i} b-$ $\left.j \Delta z) / a]^{2}\right)^{-3 / 2}$ and $Q=2 \pi / b$ with $b$ being the helix pitch and $a$ the helix radius, see Fig. 1 . The index $j$ runs along one helical turn and labels the $z$-coordinate of point charges placed along the helix. $\Delta z$ is a discretization step along the $z$-axis and the index $i$ runs over the turns of the helix and connects charges placed at sites which differ in their $z$-coordinate by a pitch length. We chose as reference $b=3.2 \mathrm{~nm}, a=1 \mathrm{~nm}$, and $\Delta z=0.32 \mathrm{~nm}$, values which are close to those of B-DNA. The factor $E_{0}$ is proportional to the local charge density and it is considered a free parameter renormalizing the bare SOC strength $\lambda$.

When coming to the electronic structure of the molecule, we will only include two molecular orbitals per molecular building block of the system. In the case of e.g. DNA, this can be the frontier molecular orbitals of the individual bases along the helix like the highestoccupied molecular orbital (HOMO) and the lowestoccupied molecular orbital (LUMO). We stress that our model is meant to be quite generic, so that we include only the minimal basic features necessary to deal with the problem in an efficient way. This is why we will not consider e.g. disorder effects related to the different base 

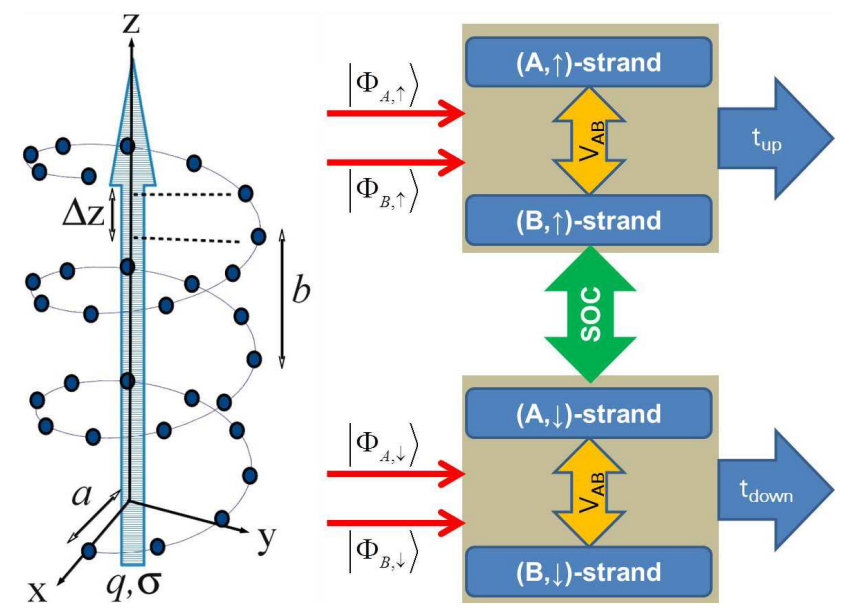

Fig. 1. Right: A schematic representation of a charge $q$ with spin $\sigma$ moving along the axis of a helical charge distribution. The parameters $a, b$ and $\Delta z$ are, respectively, radius and pitch of the helix and the spacing of the $z$-component of the position vector of the charges distributed along the helix. Left: The scattering region includes two levels $A, B$ per site in the tight-binding representation. The spin-orbit term only couples the spinup and -down channels for the same levels, i.e. there is no SOC connecting spin states on the $A$-strand with spin states on the $B$-strand. The model thus describes transport through a 4-strands ladder corresponding to the four spin-dependent channels. The imbalance between the output transmission functions $t_{\text {up }}$ and $t_{\text {down }}$ determines the spin polarization.

sequences in a real DNA molecule. Moreover, we will denote the two levels per site included in this formulation as $A, B$ to avoid a too close connection with HOMO and LUMO states.

A tight-binding model can thus be formulated and leads to the following model Hamiltonian [20]:

$$
\begin{aligned}
H & =\sum_{k=A, B} \sum_{n, \sigma} \epsilon_{n}^{k} d_{n, k, \sigma}^{\dagger} d_{n, k, \sigma} \\
& +V_{\mathrm{AB}} \sum_{n, \sigma}\left[d_{n, B, \sigma}^{\dagger} d_{n, A, \sigma}+d_{n, A, \sigma}^{\dagger} d_{n, B, \sigma}\right] \\
& +\sum_{k=A, B} \sum_{n}\left[\left(d_{n, k, \uparrow}^{\dagger}, d_{n, k, \downarrow}^{\dagger}\right) M_{n, n+1}^{k}\left(\begin{array}{c}
d_{n+1, k, \uparrow} \\
d_{n+1, k, \downarrow}
\end{array}\right)\right] \\
& +\sum_{k=A, B} \sum_{n}\left[\left(d_{n, k, \uparrow}^{\dagger}, d_{n, k, \downarrow}^{\dagger}\right) M_{n, n-1}^{k}\left(\begin{array}{c}
d_{n-1, k, \uparrow} \\
d_{n-1, k, \downarrow}
\end{array}\right)\right] .
\end{aligned}
$$

In this expression, $\epsilon_{n}^{k}=\epsilon_{k}+V_{\text {helix }}(n)+\Delta_{k}(-1)^{n}$ are site energies for the $k=A, B$ levels. They include the constant values $\epsilon_{\mathrm{A}, \mathrm{B}}$, which we set to zero for simplicity, the local contribution of the helical potential $V_{\text {helix }}(n)$ to site $n$, and we have also introduced a staggered contribution $\Delta_{k}(-1)^{n}$, which opens aband gap. The term $V_{\mathrm{AB}}$ is the local coupling between the $A$ and $B$ states on each site $n$. A gap opening can also be achieved with a large $V_{\mathrm{AB}}$; however, in order to keep the orders of magnitude of the electronic coupling parameters in a realistic range of few tens of meV, we will keep the staggered field contribution with $\Delta_{k} \sim 60 \mathrm{meV}$. The results concerning the spin polarization are not influenced qualitatively by this term. Finally, the coupling matrix $\boldsymbol{M}_{n, n \pm 1}^{k}$ has the following form:

$$
\begin{aligned}
\boldsymbol{M}_{n, n \pm 1}^{k}=\left[\begin{array}{cc}
W_{n, n \pm 1}^{k} & D_{n, n \pm 1}^{k} \\
{\left[D_{n, n \pm 1}^{k}\right]^{*}} & {\left[W_{n, n \pm 1}^{k}\right]^{*}}
\end{array}\right] \\
=\left[\begin{array}{cc}
W_{n, n \pm 1}^{k} & 0 \\
0 & {\left[W_{n, n \pm 1}^{k}\right]^{*}}
\end{array}\right]+\left[\begin{array}{cc}
0 & D_{n, n \pm 1}^{k} \\
{\left[D_{n, n \pm 1}^{k}\right]^{*}} & 0
\end{array}\right], \\
=\boldsymbol{M}_{n, n \pm 1}^{k, \mathrm{hop}}+\boldsymbol{M}_{n, n \pm 1}^{k, \mathrm{SOC}}, \\
\boldsymbol{M}_{n, n \pm 1}=\left(\boldsymbol{M}_{n \pm 1, n}\right)^{\dagger} .
\end{aligned}
$$

In Eq. (2), the diagonal blocks $W_{n, n \pm 1}^{k}$ include the (nearest-neighbor) couplings along the $A$ and $B$ pathways. We assume these quantities to be constant and site independent, so that $W_{n, n \pm 1}^{\mathrm{A}(\mathrm{B})}=V_{\mathrm{A}(\mathrm{B})}$ is the local coupling between the two levels $A$ and $B$ at site $n$. The offdiagonal blocks $D_{n, n \pm 1}^{k}$ of the $\boldsymbol{M}$-matrix couple through the SOC the up- and down-spin channels. Explicitly, these contributions look like: $D_{n, n \pm 1}^{k}=-\alpha_{\text {SOC }}^{k}(\Phi(n)+$ $\Phi(n \pm 1))$. The renormalized SOC coupling is given by $\alpha_{\text {SOC }}^{k}=\hbar E_{0}^{k} \alpha_{\text {SOC }} / 2$, where we have already included the field strength pre-factor $E_{0}$ in its definition. We have made here the assumption that the field strength may in general depend on the molecular orbital due to differences in the charge distribution associated with different orbitals, which make them to react in different ways to the helical field. As a result we get an orbital-dependent SOC effective strength [21]. We will consider from now on these new effective SOC strengths $\alpha_{\mathrm{SOC}}^{k=\mathrm{A}, \mathrm{B}}$ as free parameters. The function $\Phi(j)=\left(E_{x}(j)-\mathrm{i} E_{y}(j)\right) / E_{0}^{k}$, where the $x$ and $y$ components of the helical field are evaluated at discrete sites $z=z_{j}=j \Delta z$ along the helical axis (the $z$-axis).

This Hamiltonian in Eq. (2) preserves time reversal symmetry generated by the operator $\mathcal{T}=-i \sigma_{y} \otimes \mathcal{I}_{N \times N}$, where $N$ is the number of sites in the discretized tightbinding model and $\mathcal{I}$ is the unit matrix in the Hilbert space generated by the $N$ localized orbitals.

First-principle electronic structure calculations for e.g. DNA oligomers [27] yield values of the electronic couplings of the order of few tens of meV. We will take such orders of magnitude in our discussion below; it is clear however that to deal with a specific molecular type more careful first-principle investigations should be carried out, also including the calculation of the moleculesubstrate interaction strength.

\subsection{Charge transport}

Spin-dependent transport in the tight-binding model in Eq. (2) can be represented as a charge transport model in a 4-strand ladder model, where each strand corresponds to a molecular orbital $(A$ or $B$ ) and to a given spinor component $(\uparrow$ and $\downarrow)$. The specific transport mechanism will in general depend on the molecular system and also 
be influenced by the environmental conditions. Being aware of its potential limitations, we consider transport in the context of the Landauer approach, which provides an efficient framework to analyze the influence of different parameters on the spin polarization. The Hamiltonian (2) needs to be extended to include the coupling to the electrodes. We use a standard tunnel Hamiltonian, yielding the following additional contributions to Eq. (2):

$$
\begin{aligned}
& H_{\text {leads }}=\sum_{\beta=\mathcal{L}, \mathcal{R}} \sum_{\boldsymbol{p}} \sum_{k=A, B} \sum_{\sigma=\uparrow, \downarrow} \epsilon_{\boldsymbol{p}, k \sigma}^{\beta} c_{\beta \boldsymbol{p}, k \sigma}^{\dagger} c_{\beta \boldsymbol{p}, k \sigma} \\
& \quad+\sum_{k=A, B} \sum_{\sigma=\uparrow, \downarrow} V_{\mathcal{L} \boldsymbol{p}, k \sigma}\left(c_{\mathcal{L} \boldsymbol{p}, k \sigma}^{\dagger} d_{1, k, \sigma}+\text { H.c. }\right) \\
& +\sum_{k=A, B} \sum_{\sigma=\uparrow, \downarrow} V_{\mathcal{R} \boldsymbol{p}, \sigma}\left(c_{\mathcal{R} \boldsymbol{p}, k \sigma}^{\dagger} d_{N, k, \sigma}+\text { H.c. }\right) .
\end{aligned}
$$

Along similar lines as in Ref. [21], we consider four independent $\mathcal{L}$ (left)- and four independent $\mathcal{R}$ (right)-leads, each of them standing for a spin channel connected to a specific molecular orbital $(A, B)$ and being represented by a semi-infinite tight-binding chain. Thus, the first row describes semi-infinite tight-binding chains for each of the four independent electrodes with dispersion relation given by $\epsilon_{\boldsymbol{p}, k \sigma}^{\beta}=\epsilon_{k \sigma}^{\beta}+4 V_{0} \cos \boldsymbol{p} a$. The hopping integral $V_{0}$ is determined by diagonalizing the Hamiltonian of Eq. (2), calculating the width of its eigenvalue spectrum $\Delta E$ and taking $V_{0}=\Delta E / 4$. This choice guarantees that we include all the relevant molecular electronic states in the calculation of the transmission function. $V_{0}$ is also taken as the effective band width of the electrodes. The last two rows give the coupling between the left (right) electrode and the $n=1(n=N)$ sites of the scattering region.

The quantum mechanical transmission function is given by the standard expression: $T(E)=$ $\operatorname{Tr}\left\{G^{a}(E) \Gamma^{\mathrm{R}} G^{r}(E) \Gamma^{\mathrm{L}}\right\}$. Here $G^{r(a)}(E)$ are retarded (advanced) matrix Green's functions for the scattering region including the influence of the $H_{\text {leads }}$ via retarded self-energy matrices $\Sigma^{r, \mathcal{L}}(E)$ and $\Sigma^{r, \mathcal{R}}(E)$. The retarded Green function matrix can be determined via Dyson's equation $\left(G^{r}\right)^{-1}(E)=(E+\mathrm{i} \eta) I-H-$ $\Sigma^{r, \mathcal{L}}(E)-\Sigma^{r, \mathcal{R}}(E)$. The spectral functions $\Gamma^{\mathcal{L}, \mathcal{R}}(E)$ of the left and right electrodes are related to the selfenergies via $\Gamma^{\mathcal{L}, \mathcal{R}}(E)=-2 \operatorname{Im} \Sigma^{r, \mathcal{L}, \mathcal{R}}(E)$. The only eight non-vanishing elements of the spectral functions are $\Gamma_{\uparrow, A, B}^{\mathcal{L}}, \Gamma_{\downarrow, A, B}^{\mathcal{L}}, \Gamma_{\uparrow, A, B}^{\mathcal{R}}, \Gamma_{\downarrow, A, B}^{\mathcal{R}}$. These quantities are energy-dependent and can be computed analytically for semi-infinite chains. Using the previous expressions and approximations, the total transmission function for the system can be written as:

$$
\begin{aligned}
& T(E)=\underbrace{T_{\uparrow \uparrow}(E)+T_{\downarrow \uparrow}(E)}_{t_{\mathrm{up}}(E)}+\underbrace{T_{\uparrow \downarrow}(E)+T_{\downarrow \downarrow}(E)}_{t_{\mathrm{down}}(E)} \\
& T_{\uparrow \uparrow}(E)=\Gamma_{\uparrow, A}^{\mathcal{L}}\left[\Gamma_{\uparrow, A}^{\mathcal{R}}\left|G_{1 \uparrow, N \uparrow}\right|^{2}+\Gamma_{\uparrow, B}^{\mathcal{R}}\left|G_{1 \uparrow, 2 N \uparrow}\right|^{2}\right] \\
& \quad+\Gamma_{\uparrow, B}^{\mathcal{L}}\left[\Gamma_{\uparrow, A}^{\mathcal{R}}\left|G_{N+1 \uparrow, N \uparrow}\right|^{2}+\Gamma_{\uparrow, B}^{\mathcal{R}}\left|G_{N+1 \uparrow, 2 N \uparrow}\right|^{2}\right] \\
& T_{\uparrow \downarrow}(E)=\Gamma_{\uparrow, A}^{\mathcal{L}}\left[\Gamma_{\downarrow, A}^{\mathcal{R}}\left|G_{1 \uparrow, N \downarrow}\right|^{2}+\Gamma_{\downarrow, B}^{\mathcal{R}}\left|G_{1 \uparrow, 2 N \downarrow}\right|^{2}\right]
\end{aligned}
$$

$$
\begin{array}{r}
+\Gamma_{\uparrow, B}^{\mathcal{L}}\left[\Gamma_{\downarrow, B}^{\mathcal{R}}\left|G_{N+1 \uparrow, 2 N \downarrow}\right|^{2}+\Gamma_{\downarrow, A}^{\mathcal{R}}\left|G_{N+1 \uparrow, N \downarrow}\right|^{2}\right] \\
T_{\downarrow \downarrow}(E)=\Gamma_{\downarrow, A}^{\mathcal{L}}\left[\Gamma_{\downarrow, A}^{\mathcal{R}}\left|G_{1 \downarrow, N \downarrow}\right|^{2}+\Gamma_{\downarrow, B}^{\mathcal{R}}\left|G_{1 \downarrow, 2 N \downarrow}\right|^{2}\right] \\
+\Gamma_{\downarrow, B}^{\mathcal{L}}\left[\Gamma_{\downarrow, A}^{\mathcal{R}}\left|G_{N+1 \downarrow, N \downarrow}\right|^{2}+\Gamma_{\downarrow, B}^{\mathcal{R}}\left|G_{N+1 \downarrow, 2 N \downarrow}\right|^{2}\right] \\
T_{\downarrow \uparrow}(E)=\Gamma_{\downarrow, A}^{\mathcal{L}}\left[\Gamma_{\uparrow, A}^{\mathcal{R}}\left|G_{1 \downarrow, N \uparrow}\right|^{2}+\Gamma_{\uparrow, B}^{\mathcal{R}}\left|G_{1 \downarrow, 2 N \uparrow}\right|^{2}\right] \\
+\Gamma_{\downarrow, B}^{\mathcal{L}}\left[\Gamma_{\uparrow, B}^{\mathcal{R}}\left|G_{N+1 \downarrow, 2 N \uparrow}\right|^{2}+\Gamma_{\uparrow, A}^{\mathcal{R}}\left|G_{N+1 \downarrow, N \uparrow}\right|^{2}\right] .
\end{array}
$$

In the previous equations, $G_{n \sigma, m \nu}(E)$ with $\sigma, \nu=\uparrow, \downarrow$ are matrix elements of the previously defined retarded Green function of the SOC-active region including the influence of the $\mathcal{L}$ - and $\mathcal{R}$-electrodes via appropriate selfenergies. Each contribution in Eq. (4) can be related to a different transport process without or with spin flip. Thus, all contributions included in $T_{\uparrow \uparrow}$ and $T_{\downarrow \downarrow}$ are related to processes taking place only in the spin-up or spin-down channels, respectively, while $T_{\uparrow \downarrow}$ and $T_{\downarrow \uparrow}$ involve all processes flipping the electron spin.

The quantities $t_{\text {up }}(E)$ and $t_{\text {down }}(E)$ encode all contributions (with and without spin flip) in the outgoing spin-up and spin-down channels, respectively. With their help, we can define a spin polarization (SP) as $P(E)=\left[t_{\text {up }}(E)-t_{\text {down }}(E)\right] / T(E)$.

\section{Results}

Concerning the typical order of magnitude of the spinorbit interaction as induced by the helical field, we have provided a rough estimate of its strength in a previous study [20]; in the present investigation we are going to use similar values in the range of $3-6 \mathrm{meV} \mathrm{nm}$, being aware that a more accurate estimation would require a separate first-principle study of the electronic structure of specific molecular candidates. This may turn out computationally very demanding, mainly for molecules like DNA where the influence of the conformation and the backbones should be taken into account in an accurate way. Our goal is rather to illustrate what can be expected from a minimal approach to spin-dependent transport in a helical system. In this paper, we will keep fixed the bare electronic-coupling parameters: $V_{\mathrm{A}}=10 \mathrm{meV}$, $V_{\mathrm{B}}=90 \mathrm{meV}$ and $V_{\mathrm{AB}}=60 \mathrm{meV}$. These values have typical orders of magnitude as for a DNA molecule or other weakly $\pi$-conjugated systems. We remark, however, that we are not addressing a specific molecular system, but are deriving a generic model and investigating its main properties.

Figure 2 shows the energy-dependent spin polarization for different number of helical turns $L$. First of all, we notice that there is a rather strong tendency to negative spin polarization for hole transport (energies below the Fermi level, which is set at zero), while positive polarization at other energies is considerably weaker. Moreover, we find a tendency to odd-even effects in the global behavior of the spin polarization, with smaller values for odd lengths (left part of Fig. 2) and much larger values for even lengths (right part of the same figure). Interesting is that the dominant negative polarization has 


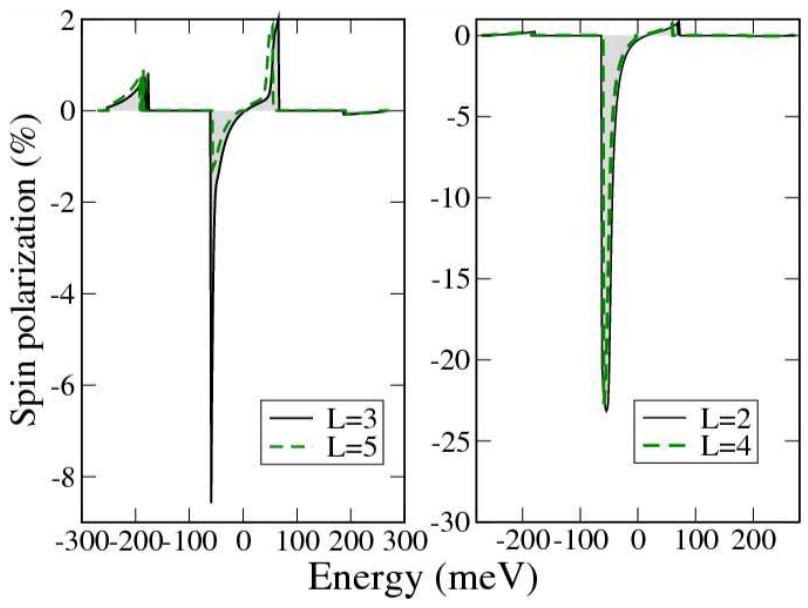

Fig. 2. Spin polarization as a function of the incoming electron energy and for different number of helical turns $L$. The incoming charge is unpolarized. A rather strong energy dependence is found, but there is a clear tendency to an oscillating behavior as a function of the number of turns between low polarization (odd $L$ ) and large polarization (even $L$ ). This effect is typical of systems where coherent tunneling dominates the transport. Electronic parameters: $V_{\mathrm{A}}=10 \mathrm{meV}, V_{\mathrm{B}}=90 \mathrm{meV}$, $V_{\mathrm{AB}}=60 \mathrm{meV}, \alpha_{\mathrm{SOC}}^{\mathrm{A}}=\alpha_{\mathrm{SOC}}^{\mathrm{B}}=6 \mathrm{meV} \mathrm{nm}$.

also been measured experimentally [7, 8]. Concerning the length dependence, the results are less conclusive: a linear dependence was found in the photo-emission experiments [7], while a less clear behavior is observed in the transport experiments [8]. The odd-even behavior we find is most likely related to a treatment based on the Landauer approach with only coherent transport included; similar odd-even effects are well-known from calculations of simple linear chains at the same level of theory [28].

Figure 3 shows the decomposition of the transmission function of the system into its individual components according to Eq. (4). The inset shows the $t_{\text {up }}$ and $t_{\text {down }}$ components of the total transmission in the outgoing spin channels. The emergence of a negative spin polarization is clearly seen in the energy window between $-60 \mathrm{meV}$ and $-40 \mathrm{meV}$, where the outgoing $t_{\text {down }}$ is clearly larger. At other energies, the difference between both components is much smaller, so that the resulting spin polarization is much weaker. The main part of Fig. 3 shows a further decomposition of $t_{\mathrm{up} \text {, down }}$ into the relative contributions of spin-flip processes $\Delta t_{\text {flip }}=T_{\uparrow \downarrow}-T_{\downarrow \uparrow}$ and nonflip processes $\Delta t_{\text {no-flip }}=T_{\uparrow \uparrow}-T_{\downarrow \downarrow}$, see also Eq. (4). From this figure, we see that both types of spin-dependent processes are contributing to the total transmissions out of the spin-up and spin-down channels. Moreover, a sizeable polarization is clearly obtained when both contributions have the same sign.

Figure 4 shows the energy dependent spin polarization for a fixed number of turns $L=4$ but with varying ratios of the orbital-dependent effective spin-orbit couplings $\alpha_{\text {SOC }}^{k}$. Notice the dramatic influence of the relative strengths, which can lead to a full suppression of the polarization in the special case $\alpha_{\mathrm{SOC}}^{\mathrm{B}} / \alpha_{\mathrm{SOC}}^{\mathrm{A}} \gg 1$.

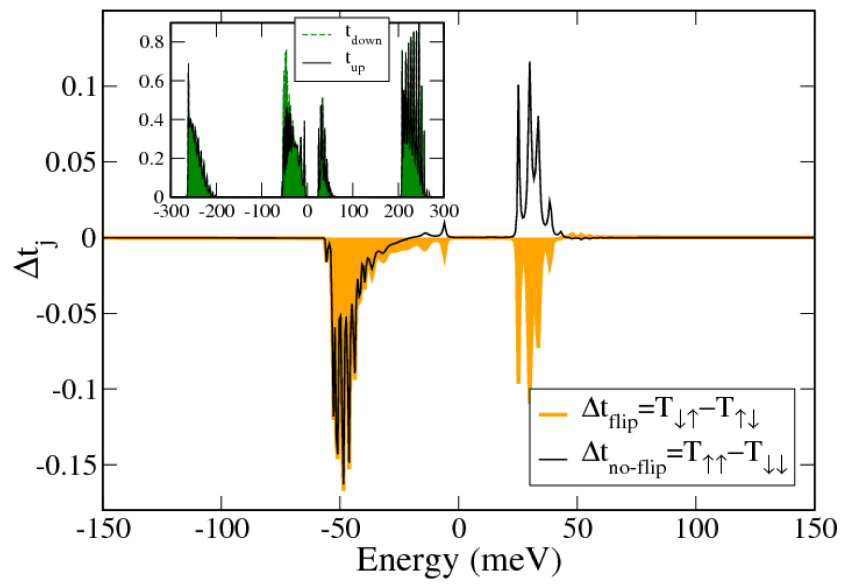

Fig. 3. Different components of the spin dependent conductance (transmission) for a fixed length of the helical domain ( $L=4$ turns, dashed line in the right part of Fig. 2). The incoming charge is unpolarized. The inset shows the total outgoing components of the spin up $\left(t_{\text {up }}\right)$ and spin down $\left(t_{\text {down }}\right)$ channels. The main panel displays a further resolution of the out going up- and down-channels into the relative contributions of spin-flip processes $\Delta t_{\text {flip }}=T_{\uparrow \downarrow}-T_{\downarrow \uparrow}$ and $\Delta t_{\text {no-flip }}=T_{\uparrow \uparrow}-T_{\downarrow \downarrow}$. The energy window around $-50 \mathrm{meV}$ where a larger spin polarization is obtained, corresponds to the case where $\operatorname{sgn}\left(t_{\text {flip }}\right)=\operatorname{sgn}\left(\Delta t_{\text {no-flip }}\right)$, compare with the right part of Fig. 2. Electronic parameters: $V_{\mathrm{A}}=10 \mathrm{meV}, V_{\mathrm{B}}=$ $90 \mathrm{meV}, V_{\mathrm{AB}}=60 \mathrm{meV}, \alpha_{\mathrm{SOC}}^{\mathrm{A}}=\alpha_{\mathrm{SOC}}^{\mathrm{B}}=6 \mathrm{meV} \mathrm{nm}$.

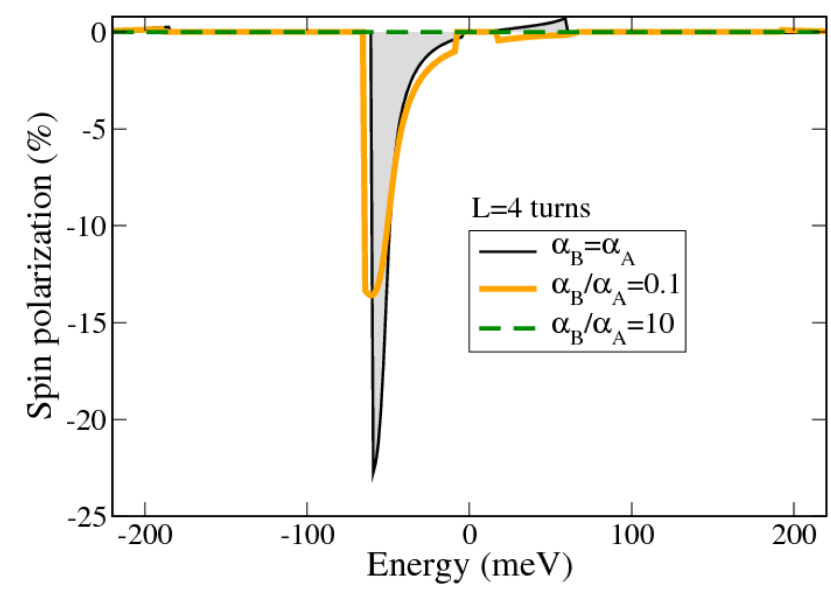

Fig. 4. Spin polarization for different ratios of the orbital-dependent effective spin-orbit strengths $\alpha_{\text {SOC }}^{k}$. For a detailed discussion of the found behavior see the main text. Electronic parameters: $V_{\mathrm{A}}=10 \mathrm{meV}$, $V_{\mathrm{B}}=90 \mathrm{meV}, V_{\mathrm{AB}}=60 \mathrm{meV}$.

We can gain a qualitative understanding of this behavior by the following analysis, which takes as starting point the continuum formulation of the tight-binding model in Eq. (2). Without going into many technical details, it can be shown that by performing a unitary transformation with the form $U_{\mathrm{A}} \oplus U_{\mathrm{B}}$ the spin-orbit 
coupling terms for the $A$ - and $B$-states can in general be removed from the Hamiltonian, as far as (i) no spindependent interactions between the states $A$ and $B$ and (ii) no electronic coupling between the states are considered $\left(V_{\mathrm{AB}}=0\right)$. This simply results from the fact that for the assumed model the spin-orbit coupling can be exactly included in the definition of the momentum as a nonAbelian gauge field, which can then be removed by an appropriate unitary transformation. The unitary operators are defined as $U_{\mathrm{k}}=\exp \left(\mathrm{i}\left(m_{k} \alpha_{\mathrm{SOC}}^{k} / \hbar\right) \int^{z} \mathrm{~d} u \boldsymbol{M}^{\mathrm{SOC}}(u)\right)$, where $\boldsymbol{M}^{\mathrm{SOC}}(u)$ is the continuum version of the spindependent tight-binding block matrix in Eq. (2). However, if e.g. $V_{\mathrm{AB}} \neq 0$ as in our case, after performing this unitary transformation the inter-level coupling $V_{\mathrm{AB}}$ is renormalized into $V_{\mathrm{AB}} \exp \left(\mathrm{i} \delta_{\mathrm{AB}} \int^{z} \mathrm{~d} u \boldsymbol{M}^{\mathrm{SOC}}(u)\right)$, with $\delta_{\mathrm{AB}}=\left(m_{\mathrm{A}} \alpha_{\mathrm{SOC}}^{\mathrm{A}} / \hbar\right)-\left(m_{\mathrm{B}} \alpha_{\mathrm{SOC}}^{\mathrm{B}} / \hbar\right)$. Since $m_{\mathrm{k}} \alpha_{\mathrm{SOC}}^{\mathrm{k}} / \hbar \sim$ $\alpha_{\mathrm{SOC}}^{k} / V_{k}$, we see that in the fully symmetric case $\alpha_{\mathrm{SOC}}^{\mathrm{A}}=$ $\alpha_{\mathrm{SOC}}^{\mathrm{B}}$ and $V_{\mathrm{A}}=V_{\mathrm{B}}$, the phase factor exactly vanishes and all spin-dependent coupling terms are removed from the Hamiltonian, so that no spin-polarization can be obtained. The same will come out in the asymmetric case, but for a combination of the SOC couplings and electronic hopping such that $\delta_{\mathrm{AB}} \ll 1$. This is the situation of the dashed line in Fig. 4. Other combinations of the ratios $\alpha_{\mathrm{SOC}}^{k} / V_{k}$ will clearly lead to further modulations of the spin polarization.

\section{Discussion and conclusions}

We have investigated within a minimal model Hamiltonian approach spin-dependent transport in a helical field. Our model assumes propagation of a charge with spin along the $z$-axis of a helical point charge distribution. The resulting helical electrostatic field leads to a spin-orbit interaction term, which resembles the Rashba SOC in semiconductors which lack inversion symmetry. However, while the standard Rashba field is usually considered a constant, the electric field here is varying in space and encodes the helical symmetry of the problem. We have shown that using this model a sizeable (mostly negative) spin polarization can be obtained. Strong energy-dependent spin polarization effects were previously demonstrated in Ref. [21] for a similar model as that presented here. The main difference was however the fact that the charge was allowed to move along a helical pathway. This change in the topology of the transport process led to diagonal spin-dependent contributions in the SOC Hamiltonian, i.e. to terms proportional to $\sigma_{z}$. Those contributions are absent in the current model, where only off-diagonal terms appear. The results of both models cannot be compared one-to-one, but it seems that the strong energy-dependence of the polarization found in Ref. [21] is strongly determined by the previously mentioned diagonal contributions to the SOC Hamiltonian, which were proportional to the curvature of the helical transport pathway and were thus strongly dependent on geometrical effects like changes in the radius of the transport pathway. The sensitivity to asymmetries in the SOC as considered here by assuming an orbitaldependent effective SOC coupling $\alpha_{\text {SOC }}^{k}$ suggest that additional studies based on a first-principle analysis of the electronic structure are required in order to make the current model more realistic. Notice also that the obtained spin polarizations do not require very large values of the SOC: it is rather the relative ratio of the SOC to the electronic coupling which seems to matter, thus further supporting the conclusions of our previous studies in Refs. [20, 21]. Further extensions of this model to describe an incoherent hopping transport scenario are currently under investigation.

\section{Acknowledgments}

RG thanks E. Díaz, C. Gaul, F. Domínguez-Adame, R. Naaman, and H. Zacharias for very fruitful discussions. This work was supported by the Volkswagen Foundation project Spintronic components based on chiral molecules (project no. 318597) and by the German Academic Exchange Service (DAAD) (project no. 54367888). This work has also been partly supported by the German Research Foundation (DFG) within the Cluster of Excellence "Center for Advancing Electronics Dresden". Computational resources were provided by the ZIH at TU-Dresden. GC thanks the NCN support No. DEC2013/10/M/ST3/00488.

\section{References}

[1] Z.H. Xiong, D. Wu, Z. Valy Vardeny, J. Shi, Nature 427, 821 (2004).

[2] S. Mooser, J.F.K. Cooper, K.K. Banger, J. Wunderlich, H. Sirringhaus, Phys. Rev. B 85, 235202 (2012).

[3] M. Grünewald, M. Wahler, F. Schumann, M. Michelfeit, C. Gould, R. Schmidt, F. Würthner, G. Schmidt, L.W. Molenkamp, Phys. Rev. B $\mathbf{8 4}$, 125208 (2011).

[4] K.-S. Li, Y.-M. Chang, S. Agilan, J.-Y. Hong, J.-C. Tai, W.-C. Chiang, K. Fukutani, P.A. Dowben, M.-T. Lin, Phys. Rev. B 83, 172404 (2011).

[5] D. Sun, L. Yin, C. Sun, H. Guo, Z. Gai, X.-G. Zhang, T.Z. Ward, Z. Cheng, J. Shen, Phys. Rev. Lett. 104, 236602 (2010).

[6] N. Atodiresei, J. Brede, P. Lazić, V. Caciuc, G. Hoffmann, R. Wiesendanger, S. Blügel, Phys. Rev. Lett. 105, 066601 (2010).

[7] B. Göhler, V. Hamelbeck, T.Z. Markus, M. Kettner, G.F. Hanne, Z. Vager, R. Naaman, H. Zacharias, Science 331, 894 (2011).

[8] Z. Xie, T.Z. Markus, S.R. Cohen, Z. Vager, R. Gutierrez, R. Naaman, Nano Lett. 11, 4652 (2011).

[9] O. Ben Dor, S. Yochelis, S.P. Mathew, R. Naaman, Y. Paltiel, Nature Commun. 4, 2256 (2013).

[10] K. Ray, S.P. Ananthavel, D.H. Waldeck, R. Naaman, Science 283, 814 (1999).

[11] I. Carmeli, V. Skakalova, R. Naaman, Z. Vager, Angew. Chem. Int. Ed. 41, 761 (2002).

[12] I. Carmeli, G. Leitus, R. Naaman, S. Reich, Z. Vager, J. Chem. Phys. 118, 10372 (2003). 
[13] Z. Vager, R. Naaman, Chem. Phys. 281, 305 (2002).

[14] R. Naaman, Z. Vager, Phys. Chem. Chem. Phys. 8, 2217 (2006).

[15] S.G. Ray, S.S. Daube, G. Leitus, Z. Vager, R. Naaman, Phys. Rev. Lett. 96, 036101 (2006).

[16] J.J. Wei, C. Schafmeister, G. Bird, A. Paul, R. Naaman, D.H. Waldeck, J. Phys. Chem. B 110, 1301 (2005).

[17] D. Mishra, T.Z. Markus, R. Naaman, M. Kettner, B. Gohler, H. Zacharias, N. Friedman, M. Sheves, C. Fontanesi, Proc. Natl. Acad. Sci. USA 110, 14872 (2013).

[18] S. Yeganeh, M.A. Ratner, E. Medina, V. Mujica, J. Chem. Phys. 131, 014707 (2009).

[19] E. Medina, F. Lopez, M. Ratner, V. Mujica, Eur. Phys. Lett. 99, 17006 (2012).

[20] R. Gutierrez, E. Díaz, R. Naaman, G. Cuniberti, Phys. Rev. B 85, 081404 (2012).

[21] R. Gutierrez, E. Díaz, C. Gaul, T. Brumme, F. Domínguez-Adame, G. Cuniberti, J. Phys. Chem. C 117, 22276 (2013).
[22] A.M. Guo, Q.F. Sun, Phys. Rev. Lett. 108, 218102 (2012).

[23] J. Gersten, K. Kaasbjerg, A. Nitzan, J. Chem. Phys. 139, 114111 (2013).

[24] A.A. Eremko, V.M. Loktev, Phys. Rev. B 88, 165409 (2013).

[25] A.-M. Guo, E. Díaz, C. Gaul, R. Gutierrez, F. Domínguez-Adame, G. Cuniberti, Q.F. Sun, Phys. Rev. B 89, 205434 (2014).

[26] D. Hochberg, G. Edwards, T.W. Kephart, Phys. Rev. E 55, 3765 (1997).

[27] T. Kubar, P.B. Woiczikowski, G. Cuniberti, M. Elstner, J. Phys. Chem. B 112, 7937 (2008).

[28] R. Gutiérrez, F. Grossmann, O. Knospe, R. Schmidt, Phys. Rev. A 64, 013202 (2001). 